\title{
Irreversible evolution of a wave packet in the rigged-Hilbert-space quantum mechanics
}

\author{
Giulia Marcucci $^{*}$ and Claudio Conti ${ }^{\dagger}$ \\ Institute for Complex Systems, National Research Council (ISC-CNR), Via dei Taurini 19, 00185 Rome, Italy \\ and Department of Physics, University Sapienza, Piazzale Aldo Moro 5, 00185 Rome, Italy
}

(Received 11 August 2016; published 29 November 2016)

\begin{abstract}
It is well known that a state with complex energy cannot be the eigenstate of a self-adjoint operator, such as the Hamiltonian. Resonances, i.e., states with exponentially decaying observables, are not vectors belonging to the conventional Hilbert space. One can describe these resonances in an unusual mathematical formalism based on the so-called rigged Hilbert space (RHS). In the RHS, the states with complex energy are denoted as Gamow vectors $(\mathrm{GVs})$, and they model decay processes. We study the GVs of the reversed harmonic oscillator, and we analytically and numerically investigate the unstable evolution of wave packets. We introduce the background function to study initial data that are not composed only by a summation of GVs, and we analyze different wave packets belonging to specific function spaces. Our work furnishes support for the idea that irreversible wave propagation can be investigated using rigged-Hilbert-space quantum mechanics and provides insight for the experimental investigation of irreversible dynamics.
\end{abstract}

DOI: 10.1103/PhysRevA.94.052136

\section{INTRODUCTION}

The spontaneous decay of nuclear particles, or the empirical evidence for the Big Bang, has led various authors [1-6] to consider modifications of the principles of quantum mechanics (QM) in order to include time asymmetry. Despite a long-standing effort, the need for these modifications is still debated. However, the debate has stimulated the development of theoretical tools and paradigms by a growing community of scientists. These tools have recently found surprising applications in nonlinear physics and photonics [7-9].

The time-asymmetric dynamics of quantum systems are also relevant in biophysics, fluid dynamics, network theory, entanglement generation, and epigenetic studies (see, for example, [10-12] and the references therein). The decay from local maxima in the energetic landscape of complex systems is generically retained irreversible. However, the origin of this irreversibility in the case of microscopic quantum structures or in quantum-inspired models of networks [13] is unconsidered so far [14].

The leading theoretical background of time-asymmetric quantum mechanics (TA-QM) is the rigged Hilbert space (RHS), an enlarged Hilbert space that includes nonnormalizable wave packets that get amplified or decay exponentially with time. The paradigmatic model for TA-QM is the reversed harmonic oscillator (RHO). Within standard QM the description of the evolution of a wave function may be done by using the continuous spectrum of the Hamiltonian. In TA-QM one considers a RHS, and generalized eigenvalues with complex energies do have physical meanings [15]. The corresponding non-normalizable eigenvectors are the so-called Gamow vectors (GVs) [16,17] and they form a numerable generalized basis for integrable functions. This discrete summation of the continuous spectrum furnishes novel physical insights for the spontaneous decay of a wave packet. For example, one can predict the surprising result of the

\footnotetext{
*marcucci.giulia@gmail.com
}

†http://www.complexlight.org quantization of the decay rates, which has been experimentally observed in an optical emulation $[8,18]$.

A further intriguing outcome of the TA-QM is the fact that it unveils a particular structure in the phase space. Namely, one can discriminate initial data in terms of their projection on the eigenvectors of the RHO continuous spectrum. These projections engender different vector subspaces depending on specific Hardy spaces. The question of whether this classification has a direct physical counterpart has no answer at the moment, albeit mathematically this has important implications. If the initial wave function belongs to a particular space, it is completely represented by a numerable set of generalized eigenfunctions. On the contrary, the representation is given in terms of a finite number of $\mathrm{GVs}$ and a background function.

Despite that these mathematical properties have been studied by several authors [19-26], a direct physical evidence of their implications is lacking, even in the simplest case of RHO. In this paper, we review the basics of TA-QM and of the GV approach to the RHO. Moreover, we study the way the function space of the input wave functions has a direct counterpart in the long-term evolution, also using numerical methods [27] to show similarities and differences in the behavior.

This article is organized as follows. In Sec. II we present three theorems which establish that quantum mechanics with a temporal asymmetry cannot exist in the standard Hilbert-space formulation. In Sec. III we build a different topology for the space of initial data and show the way this new geometry causes an enlargement of the Hilbert space, namely, the rigged Hilbert space. Direct consequences of RHS are disclosed in Sec. IV. In Sec. V we describe quantized damped motion, and in Sec. VI we study RHO. In Secs. VII and VIII we study the evolution of a function with compact support and a Gaussian function, respectively. We show the differences between their propagation: since the first one belongs to a specific function space, it exponentially decays with time; in contrast, since the Gaussian function does not have a compact support, it is the superposition of exponentially and algebraically decaying waves. Conclusions are drawn in Sec. IX. 


\section{FUNDAMENTAL THEOREMS OF QM}

To build a mathematical theory behind a generic quantum system, we need to define a Hausdorff vector space $\Psi$ with a locally convex topology $\tau$ and a scalar product $(\cdot \mid \cdot)$. We need also an algebra $\mathcal{A}$ of $\tau$-continuous linear operator on $\Psi$ and a probability measure $\mathcal{P}$ on $\mathcal{A}$. By the scalar product $(\cdot \mid \cdot)$, we are able to build a norm $\|\psi\|=\sqrt{(\psi \mid \psi)} \forall \psi \in \Psi$ and a metric $d(\psi, \phi)=\|\phi-\psi\| \forall \phi, \psi \in \Psi$, which is induced by the norm, therefore we can settle a new topology $\tau_{d}$ on $\Psi$, given by the distance $d$. Now, we have a Euclidean space $\left(\Psi, \tau_{d}\right)$, which is also normed and separable. To be a physical space it needs the completeness.

Let $\left(\mathcal{H}, \tau_{\mathcal{H}}\right)$ be the completion of $\left(\Psi, \tau_{d}\right) ; \mathcal{H}$ is a separable Hilbert space and is the space used to formulate the known time-symmetric quantum theory. The temporal symmetry in a Hilbert space arises from the following three theorems:

Theorem II.1 (Gleason) [28]. For every probability $\mathcal{P}(\Lambda)$, there exists a positive trace class operator $\rho$ such that

$$
\mathcal{P}(\Lambda)=\operatorname{Tr}(\Lambda \rho) .
$$

Theorem II.2 (Stone-Neumann) [29]. Let us consider the Schrödinger-Neumann equation for $\rho$ previously defined

$$
\frac{\partial \rho(t)}{\partial t}=\frac{i}{\hbar}[H, \rho(t)],
$$

with $H$ the Hamiltonian operator. The solutions of such an equation are time symmetric and they are given by the group of unitary operators $U^{\dagger}(t)=\exp \left(-\frac{i}{\hbar} H t\right)$.

Theorem II.3 (Hegerfeldt) [30]. For every Hermitian and semi-bounded Hamiltonian $H$, either

$$
\operatorname{Tr}[\Lambda(t) \rho]=\operatorname{Tr}[\Lambda \rho(t)]=0 \forall t \in \mathbb{R}
$$

or

$$
\operatorname{Tr}[\Lambda(t) \rho]=\operatorname{Tr}[\Lambda \rho(t)]>0 \quad \forall t \in \mathbb{R},
$$

except on a set of Lebesgue measure zero.

These theorems imply that time-asymmetric solutions of the Schrödinger equation

$$
i \hbar \frac{\partial \phi(t)}{\partial t}=H \phi(t)
$$

with time-asymmetric boundary conditions are not allowed, hence we need to modify the mathematical description of the system.

\section{RIGGED-HILBERT-SPACE TOPOLOGY}

For every fixed $\psi_{0} \in \Psi$, the translation $T: \Psi \rightarrow \Psi$ such that $\psi \rightarrow \psi+\psi_{0}$ is a linear homeomorphism of $\Psi$ on itself. Therefore $\tau$ is uniquely determined by the neighborhood system $I(0)$ centered at the origin, because every other neighborhood of any point $\psi$ of $\Psi$ is obtained by translating a neighborhood of the origin of the vector $\psi .(\Psi, \tau)$ is said to be locally convex if $\mathcal{C}=\{C \in I(0) \mid C$ is convex $\}$ is a neighborhood local basis. Since every open ball centered at the origin is convex, it is also a member of $\mathcal{C}$ if and only if $\exists A \in \tau \mid 0 \in A \subset B_{r}(0) \forall B_{r}(0)$. By this last condition, we build a locally convex topology $\tau$ on $\Psi$ that is finer than the topology $\tau_{d}$ induced by the norm.
Let us suppose that $(\Psi, \tau)$ and $\left(\mathcal{H}, \tau_{\mathcal{H}}\right)$ are the previously described spaces and, besides, $\tau$ is locally convex and finer than $\tau_{\mathcal{H}}$. Then we can define another completion $\Phi$ of $\Psi$, this time with respect to $\tau$, and find another complete space $\left(\Phi, \tau_{\Phi}\right)$ that is different from $\left(\mathcal{H}, \tau_{\mathcal{H}}\right)$. Precisely, $\Phi \subset \mathcal{H}$, and $\Phi$ is dense in $\mathcal{H}$. Moreover, $\Phi \subset \mathcal{H} \Rightarrow \mathcal{H}^{*} \subset \Phi^{*}$, where $\mathcal{H}^{*}$ and $\Phi^{*}$ are the dual spaces of $\mathcal{H}$ and $\Phi$, respectively.

The definition of dual space is the basis for building a RHS and we need a more physically accessible dual space, according to $[1,2]$. Let $\mathcal{E}$ be a Euclidean space. We identify the scalar product on $\mathcal{E}$ as $(\cdot \mid \cdot)$; instead $\langle\cdot \mid \cdot\rangle$ is the operatorial product on the dual space $\mathcal{E}^{*}$, namely, $F(v)=\langle F \mid v\rangle$. We define our dual space $\Phi^{\times}$as the space of antilinear and continuous functionals on $\Phi$, that is,

$$
F \in \Phi^{\times} \Longleftrightarrow F(\phi)=\langle\phi \mid F\rangle .
$$

Thus every functional in $\Phi^{\times}$has a sort of complex conjugate in $\Phi^{*}$, and the Riesz-Frechet representation theorem on the Hilbert space $\mathcal{H}$ still works, hence $\mathcal{H}=\mathcal{H}^{\times}$. In this manner we obtain the Gelfand triplet $\Phi \subset \mathcal{H} \subset \Phi^{\times}$, which defines our RHS.

\section{GAMOW VECTORS}

It is well known that in order to be observable, the Hamiltonian operator $H$ of a quantum system must be selfadjoint on $\mathcal{H}$, so $H=H^{\dagger}$. Nevertheless $H \neq H^{\times}$on $\Phi^{\times}$.

Let us consider the secular equation

$$
H^{\times}|E\rangle=E|E\rangle \text {. }
$$

If $|E\rangle \in \Phi^{\times} \backslash \mathcal{H}$, we cannot affirm that the corresponding eigenvalue $E$ is a real number. We define a generalized eigenvector $|E\rangle \in \Phi^{\times}$, which has a complex eigenvalue, as a Gamow vector $\left|\phi_{G}\right\rangle=\left|E^{ \pm}\right\rangle=\left|E_{R} \pm i \frac{\Gamma}{2}\right\rangle$ (subscript $R$ is due to one of the first applications of this theory, which Bohm developed in scattering experiments [1], and it is related to the resonances of the system). From the Schrödinger equation (in units such that $\hbar=1$ ), we get a unitary operator $U(t)=e^{-i H t}$ for the temporal evolution of any state in $\mathcal{H}$. We see that $U(t)^{\times}=e^{i H^{\times} t}$ is not unitary on $\Phi^{\times}$:

$$
U(t)^{\times}\left|E_{R} \pm i \frac{\Gamma_{n}}{2}\right\rangle=e^{i E_{R} t} e^{\mp \frac{\Gamma_{n}}{2} t}\left|E_{R} \pm i \frac{\Gamma_{n}}{2}\right\rangle .
$$

$U(t)^{\times}$is not an isometry, because

$$
\| U(t)^{\times}\left|E_{R} \pm i \frac{\Gamma_{n}}{2}\right\rangle\left\|^{2}=e^{\mp \Gamma_{n} t}\right\|\left|E_{R} \pm i \frac{\Gamma_{n}}{2}\right\rangle \|^{2} .
$$

Moreover

$$
\| U(t)^{\times}\left|E_{R} \pm i \frac{\Gamma_{n}}{2}\right\rangle \stackrel{t \rightarrow \pm \infty}{\longrightarrow} 0,
$$

and

$$
\| U(t)^{\times}\left|E_{R} \pm i \frac{\Gamma_{n}}{2}\right\rangle \| \stackrel{t \rightarrow \mp \infty}{\longrightarrow}+\infty .
$$

In a physical context, we need to identify $\Phi$ with the Schwartz space $S\left(\mathbb{R}^{N}\right)$, that is, the space of rapidly decreasing functions, and the Hilbert space $\mathcal{H}$ with the space of quadratically integrable functions $\mathcal{L}^{2}\left(\mathbb{R}^{N}\right)$, so these last two 
expressions suggest that we need to define the following new spaces:

$$
\begin{aligned}
& \Phi_{-}=\left\{\phi \in \Phi \mid f(E)=\left\langle\phi \mid E^{-}\right\rangle \in S(\mathbb{R}) \cap \mathcal{H}_{-}^{2}\right\}, \\
& \Phi_{+}=\left\{\phi \in \Phi \mid f(E)=\left\langle\phi \mid E^{+}\right\rangle \in S(\mathbb{R}) \cap \mathcal{H}_{+}^{2}\right\} ;
\end{aligned}
$$

where $\mathcal{H}_{-}^{2}$ and $\mathcal{H}_{+}^{2}$ are Hardy spaces bounded from below and from above, respectively. To sum up, $\Phi_{ \pm}$are dense in $\Phi$, $\Phi=\Phi_{-}+\Phi_{+}\left(\Phi_{-} \cap \Phi_{+} \neq 0\right.$ generally $)$, and $\Phi$ is dense in $\mathcal{H}$, consequently,

$$
\begin{aligned}
& \Phi_{-} \stackrel{\text { dense }}{\complement} \Phi \stackrel{\text { dense }}{\subset} \mathcal{H} \stackrel{\text { dense }}{\complement} \Phi^{\times} \stackrel{\text { dense }}{\complement} \Phi_{-}^{\times}, \\
& \Phi_{+} \stackrel{\text { dense }}{\complement} \Phi \stackrel{\text { dense }}{\complement} \mathcal{H} \stackrel{\text { dense }}{\complement} \Phi^{\times} \stackrel{\text { dense }}{\complement} \Phi_{+}^{\times} .
\end{aligned}
$$

We have now found two Gelfand triplets, $\Phi_{-} \subset \mathcal{H} \subset \Phi_{-}^{\times}$ and $\Phi_{+} \subset \mathcal{H} \subset \Phi_{+}^{\times}$, where the evolution operator $U(t)$ acts as a semigroup, because it is well defined and continuous only for $t \leqslant 0$ on $\Phi_{-}$, and only for $t \geqslant 0$ on $\Phi_{+}$. The value $t=0$ expresses the intrinsic irreversibility we have when, for example, we divide an experiment into a preparation stage and a registration stage. In this case, $\Phi_{-}$will be the space of the initial states and $\Phi_{+}$will be the space of the detected states.

\section{QUANTIZATION OF A DAMPED MOTION}

For its simplicity and its relevance, the harmonic oscillator (HO) can be chosen to introduce the study of quantum mechanics in a time-symmetric context $[19,20]$. The classical HO Hamiltonian is

$$
H=\frac{p^{2}}{2 m}+\frac{m \omega^{2}}{2} x^{2} .
$$

We quantize the HO by converting the canonical coordinates $x, p$ into the operators $\hat{x}, \hat{p}$ such that

$$
[\hat{x}, \hat{p}]=i \hbar,
$$

and we find the spectrum of $H$ :

$$
\begin{aligned}
H \psi(x) & =E \psi(x), \quad E_{n}=\hbar \omega\left(n+\frac{1}{2}\right), \\
\psi_{n}(x) & =\sqrt[4]{\frac{m \omega}{\hbar \pi}} \frac{1}{\sqrt{2^{n} n !}} H_{n}\left(\sqrt{\frac{m \omega}{\hbar}} x\right),
\end{aligned}
$$

where $H_{n}(x)=(-1)^{n} x^{2} \frac{d^{n}}{d x^{n}} e^{-x^{2}}$ are the Hermite polynomials.

In a time-asymmetric context, considering the equation of a damped motion comes natural for its inherent irreversibility. In fact, if we consider the classical dynamical system in one dimension

$$
\begin{aligned}
\frac{d}{d t} u(t) & =-\gamma u(t), \\
u(0) & =u_{0},
\end{aligned}
$$

where $\gamma>0$ and $m=\hbar=1$, we have

$$
u(t)=e^{-\gamma t} u_{0},
$$

which represents a damping for $t \geqslant 0$. We quantize it exactly as we did for the $\mathrm{HO}$, even if this one is not a Hamiltonian system. In a general $n$-dimensional space, one defines a dynamical system as

$$
\frac{d u}{d t}=X(u),
$$

where $X$ is a vector field. Using canonical coordinates $\left(u^{1}, \ldots, u^{n}, v^{1}, \ldots, v^{n}\right)$, we get the Hamiltonian

$$
H(u, v)=\sum_{k=1}^{n} v_{k} X_{k}(u),
$$

where $X_{k}$ are the components of $X$ in the coordinate basis, so for Eq. (9)

$$
H(u, v)=-\gamma u v .
$$

Since the quantization must take into account that $\hat{v}$ does not commute with $\hat{u}$, we have

$$
\hat{H}(\hat{u}, \hat{v})=-\frac{\gamma}{2}(\hat{u} \hat{v}+\hat{v} \hat{u}) .
$$

By performing the canonical transformation

$$
\hat{u}=\frac{\gamma \hat{x}-\hat{p}}{\sqrt{2 \gamma}}, \quad \hat{v}=\frac{\gamma \hat{x}+\hat{p}}{\sqrt{2 \gamma}},
$$

one obtains the Hamiltonian of the reversed harmonic oscillator (RHO):

$$
\hat{H}(\hat{x}, \hat{p})=\frac{\hat{p}^{2}}{2}-\frac{\gamma^{2} \hat{x}^{2}}{2} .
$$

Let us compare $\mathrm{HO}$ and $\mathrm{RHO}$. We pass from the first one to the second one by changing $\omega$ into the complex value $i \gamma$ [25]. This simple transformation allows us to move from a parabolic potential bounded from below to a parabolic barrier. This potential overturning produces a completely different physics: the $\mathrm{HO}$ models the behavior of a pointlike mass around a stable equilibrium and the RHO gives the dynamics around an unstable equilibrium, an intrinsically irreversible evolution (at variance with an oscillator, a falling body never goes back to its initial position).

In this section, we analyze the Hamiltonian of the damped motion, defined in Eq. (10). As proved in [19], $\hat{H}(\hat{u}, \hat{v})$ is self-adjoint on $\mathcal{L}^{2}(\mathbb{R})$ and parity invariant. We define the timereversal operator $T$ such that

$T \phi(t):=\phi(-t) \Rightarrow T U(t)=U^{\dagger}(t) T \Rightarrow U(t) T U(t)=T$,

where $U(t):=e^{-i H t}$. $T$ plays a fundamental role in this system, and coincides with the inverse Fourier transformation, i.e., $T \phi(u, t):=\check{F}[\phi](u, t)$, where

$$
\check{F}[\phi](x, t)=\frac{1}{\sqrt{2 \pi}} \int_{\mathbb{R}} e^{i k x} \phi(k, t) d k .
$$

Let us define two families of tempered distributions in $\Phi^{\times}$, the first one

$$
\begin{aligned}
\hat{u}\left|f_{0}^{-}\right\rangle & :=0, \quad f_{0}^{-}(u)=\delta(u), \\
\forall n \in \mathbb{N} \quad\left|f_{n}^{-}\right\rangle: & =\frac{(-i)^{n}}{\sqrt{n !}} \hat{v}^{n}\left|f_{0}^{-}\right\rangle \\
\Rightarrow \quad f_{n}^{-}(u) & =\frac{(-1)^{n}}{\sqrt{n !}} \frac{d^{n}}{d u^{n}} \delta(u) ;
\end{aligned}
$$


and the second one

$$
\begin{aligned}
& \hat{v}\left|f_{0}^{+}\right\rangle:=0, \quad f_{0}^{+}(u)=1, \\
& \forall n \in \mathbb{N} \quad\left|f_{n}^{+}\right\rangle:=\frac{1}{\sqrt{n !}} \hat{u}^{n}\left|f_{0}^{+}\right\rangle \Rightarrow f_{n}^{+}(u)=\frac{u^{n}}{\sqrt{n !}} .
\end{aligned}
$$

Hereafter, following [1-3], we denote a tempered distribution $f_{n}^{ \pm}$a resonance. We can see that

$$
H^{\times}\left|f_{n}^{ \pm}\right\rangle= \pm E_{n}\left|f_{n}^{ \pm}\right\rangle,
$$

where $E_{n}:=i \gamma\left(n+\frac{1}{2}\right) \in \mathbb{C}$. Given that $f_{n}^{ \pm}$are tempered distributions, their inverse Fourier transforms are well defined, and they are

$$
\begin{gathered}
\check{F}\left[f_{n}^{-}\right]=\frac{i^{n}}{\sqrt{2 \pi}} f_{n}^{+}, \\
\check{F}\left[f_{n}^{+}\right]=i^{n} \sqrt{2 \pi} f_{n}^{-} .
\end{gathered}
$$

We show the quasiorthogonality and the quasicompleteness of the resonances:

$$
\begin{aligned}
\left\langle f_{n}^{-} \mid f_{m}^{+}\right\rangle & =\delta_{n, m}, \\
\sum_{n=0}^{\infty} f_{n}^{-}(u) f_{n}^{+}(y) & =\delta(u-y) .
\end{aligned}
$$

To find real energy values, we need to analyze also the continuous spectrum. Since $H$ is parity invariant, each generalized eigenvalue is doubly degenerate, thus

$$
H^{\times} \psi_{ \pm}^{E}=E \psi_{ \pm}^{E} .
$$

As one can see in [19], the generalized eigenfunctions are

$$
\psi_{ \pm}^{E}(u)=\frac{1}{\sqrt{2 \pi \gamma}} u_{ \pm}^{-\left(\frac{i E}{\gamma}+\frac{1}{2}\right)},
$$

where $u_{ \pm}^{\lambda}$ are tempered distributions such that

$$
\begin{aligned}
& u_{+}^{\lambda}:=\left\{\begin{array}{cc}
u^{\lambda} & u \geqslant 0, \\
0 & u<0,
\end{array}\right. \\
& u_{-}^{\lambda}:=\left\{\begin{array}{cc}
0 & u<0, \\
u^{\lambda} & u \leqslant 0 .
\end{array}\right.
\end{aligned}
$$

It is possible to prove both the orthonormality and the completeness of the eigenfunctions, namely,

$$
\begin{aligned}
& \sum_{ \pm} \int\left[\psi_{ \pm}^{E_{1}}(u)\right]^{*} \psi_{ \pm}^{E_{2}}(u) d u=\delta\left(E_{1}-E_{2}\right), \\
& \sum_{ \pm} \int\left[\psi_{ \pm}^{E}(u)\right]^{*} \psi_{ \pm}^{E}\left(u^{\prime}\right) d E=\delta\left(u-u^{\prime}\right) .
\end{aligned}
$$

Therefore we can apply the Gelfand-Maurin theorem [24] and write any function in $S(\mathbb{R})$ as

$$
\phi(u)=\sum_{ \pm} \int \psi_{ \pm}^{E}(u)\left\langle\phi \mid \psi_{ \pm}^{E}\right\rangle^{*} d E .
$$

By repeating the same reasoning,

$$
H^{\times} \check{F}\left[\psi_{ \pm}^{-E}\right]=E \check{F}\left[\psi_{ \pm}^{-E}\right],
$$

so one can prove also the orthonormality and the completeness of the inverse Fourier transforms of the eigenfunctions, whence

$$
\phi(u)=\sum_{ \pm} \int \check{F}\left[\psi_{ \pm}^{-E}\right](u)\left\langle\phi \mid \check{F}\left[\psi_{ \pm}^{-E}\right]\right\rangle^{*} d E .
$$

We have just defined two groups of eigenfunctions, $\psi_{ \pm}^{E}(u)$ and $\check{F}\left[\psi_{ \pm}^{-E}\right](u)$, which represent the continuous spectrum of the Hamiltonian of a damped motion into the RHS. Moreover, we have just seen that they depend on the tempered distributions $u_{ \pm}^{-\left(\frac{i E}{\gamma}+\frac{1}{2}\right)}$, which have simple poles in the complex plane when

$$
E=-E_{n}=-i \gamma\left(n+\frac{1}{2}\right) .
$$

Thanks to the properties of the generalized function $u_{ \pm}^{\lambda}$ [19], we can finally state what follows:

$$
\begin{gathered}
\operatorname{Res}\left[\psi_{ \pm}^{E},-E_{n}\right]=\frac{( \pm 1)^{n} i \sqrt{\gamma}}{\sqrt{2 \pi n !}} f_{n}^{-}, \\
\operatorname{Res}\left[\check{F}\left[\psi_{ \pm}^{-E}\right], E_{n}\right]=\frac{( \pm i)^{n} i \sqrt{\gamma}}{2 \pi \sqrt{n !}} f_{n}^{+} .
\end{gathered}
$$

By defining the following spaces, we get two Gelfand triplets:

$$
\begin{gathered}
\mathcal{H}=\mathcal{L}^{2}(\mathbb{R}), \quad \Phi=S(\mathbb{R}), \\
\Phi_{-}=\left\{\phi \in \Phi \mid f(E)=\left\langle\phi \mid \check{F}\left[\psi_{ \pm}^{-E}\right]\right\rangle \in \mathcal{H}_{-}^{2}\right\}, \\
\Phi_{+}=\left\{\phi \in \Phi \mid f(E)=\left\langle\phi \mid \psi_{ \pm}^{E}\right\rangle \in \mathcal{H}_{+}^{2}\right\} .
\end{gathered}
$$

From this framework into the RHS $\Phi^{\times}$, we can infer the irreversible evolution of certain waves in $\Phi$. We established above the connection between the continuous and the point spectra. Now we make this link definitively clear and we show that the evolution operator acts as a semigroup on $\Phi_{ \pm}$for a well-defined orientation of the arrow of time. By recalling Eqs. (20) and (21), we apply the residue theorem to initial data in $\Phi_{ \pm}$[19] and get two different expansions in GVs:

$$
\begin{aligned}
\phi^{+}(u) & =\sum_{n=0}^{+\infty}\left\langle\phi^{+} \mid f_{n}^{+}\right\rangle f_{n}^{-}(u) \forall \phi^{+} \in \Phi_{+}, \\
\phi^{-}(u) & =\sum_{n=0}^{+\infty}\left\langle\phi^{-} \mid f_{n}^{-}\right\rangle f_{n}^{+}(u) \forall \phi^{-} \in \Phi_{-} .
\end{aligned}
$$

Thanks to the following definitions of two new function spaces, both of them subspaces of $S(\mathbb{R})$ and isomorphic by the inverse Fourier transformation, we can establish the relation between $\Phi_{+}$and $\Phi_{-}: \mathcal{D}=C_{c}^{\infty}(\mathbb{R})$ is the space of the infinitely differentiable functions with compact support; $\mathcal{Z}=$ $\{\check{F}[\phi] \mid \phi \in \mathcal{D}\}$, where $\check{F}$ is the inverse Fourier transformation. Since for each function $\phi \in \mathcal{Z}$, we have

$$
\phi(u)=\left.\sum_{n=0}^{+\infty} \frac{1}{n !} \frac{d^{n}}{d u^{n}} \phi(u)\right|_{u=0} u^{n}=\sum_{n=0}^{+\infty} f_{n}^{+}(u)\left\langle f_{n}^{-} \mid \phi\right\rangle,
$$

while, at the same time, every $\psi \in \mathcal{D}$ is the Fourier transform of a function in $\mathcal{Z}$, hence

$$
\psi(u)=\frac{1}{\sqrt{2 \pi}} \int_{\mathbb{R}} \check{F}[\psi](v) e^{-i v u} d v=\sum_{n=0}^{+\infty} f_{n}^{-}(u)\left\langle f_{n}^{+} \mid \psi\right\rangle .
$$


We can state that

$$
\Phi_{+} \equiv \mathcal{D}, \quad \Phi_{-} \equiv \mathcal{Z} .
$$

At last, we study the evolution operator $U(t)=e^{-i H t} . U$ is a unitary group on $\mathcal{H}=\mathcal{L}^{2}(\mathbb{R})$, given that if $\psi(u, 0) \in \mathcal{L}^{2}(\mathbb{R})$ then

$$
\psi(u, t)=U(t) \psi(u, 0)=e^{\frac{\gamma}{2} t} \psi\left(e^{\gamma t} u, 0\right),
$$

a transformation that turns out to be an isometry on $\mathcal{L}^{2}(\mathbb{R})$. This means that if $\psi(u, t)$ solves the Schrödinger equation, then also $T \psi(u, t)=\psi(u,-t)$ does. Therefore the theory is time-reversal invariant on the Hilbert space $\mathcal{H}$, without letting us see the damping we expected. Where do we observe the temporal irreversibility? It lacks the analysis of $U$ restricted to $\Phi_{ \pm}$. If $\phi^{+}(u, 0) \in \Phi_{+}$then

$$
\begin{aligned}
\left\langle U(t) \phi^{+} \mid \psi_{ \pm}^{E}\right\rangle & =\left\langle\phi^{+} \mid U^{\times}(t) \psi_{ \pm}^{E}\right\rangle \\
& =e^{i E t}\left\langle\phi^{+} \mid \psi_{ \pm}^{E}\right\rangle \in \mathcal{H}_{+}^{2} \Leftrightarrow t \geqslant 0 ;
\end{aligned}
$$

on the other hand, if $\phi^{-}(u, 0) \in \Phi_{-}$then

$$
\begin{aligned}
\left\langle U(t) \phi^{-} \mid \check{F}\left[\psi_{ \pm}^{-E}\right]\right\rangle & =\left\langle U(-t) \check{F}\left[\phi^{-}\right] \mid \psi_{ \pm}^{-E}\right\rangle \\
=\left\langle\check{F}\left[\phi^{+}\right] \mid U^{\times}(-t) \psi_{ \pm}^{-E}\right\rangle & =e^{i E t}\left\langle\check{F}\left[\phi^{-}\right] \mid \psi_{ \pm}^{-E}\right\rangle \\
=e^{i E t}\left\langle\phi^{-} \mid \check{F}\left[\psi_{ \pm}^{-E}\right]\right\rangle & \in \mathcal{H}_{-}^{2} \Leftrightarrow t \leqslant 0 .
\end{aligned}
$$

We conclude that $U(t)$ establishes two semigroups:

$$
U_{+}(t): \Phi_{+} \longrightarrow \Phi_{+} \forall t \geqslant 0
$$

and

$$
U_{-}(t): \Phi_{-} \longrightarrow \Phi_{-} \forall t \leqslant 0 .
$$

We have just found a way to model irreversible phenomena. In fact, the action of $U$ allows us to choose an orientation of the temporal arrow: if it goes forward from zero, then our initial data are in $\Phi_{+}$, otherwise they are in $\Phi_{-}$, indeed,

$$
\phi^{+}(u, t)=\sum_{n} e^{-\gamma(n+1 / 2) t}\left\langle\phi^{+} \mid f_{n}^{+}\right\rangle f_{n}^{-}(u)
$$

and

$$
\phi^{-}(u, t)=\sum_{n} e^{\gamma(n+1 / 2) t}\left\langle\phi^{-} \mid f_{n}^{-}\right\rangle f_{n}^{+}(u) .
$$

Moreover, all the physics we get fixing a specific orientation of time's arrow is achievable fixing the other one too, because the time-reversal operator $T$ establishes an isomorphism between $\Phi_{+}$and $\Phi_{-}$, in fact,

$$
T \phi^{+}(u, t)=U(-t) T \phi^{+}(u, 0)=\phi^{-}(u,-t) .
$$

Summarizing, we obtained an irreversible quantum system by observing that the evolution operator acts as a semigroup on $\Phi_{ \pm}$, due to the presence of resonant states $f_{n}^{ \pm}$. In this way, the instant $t=0$ separates the evolution in two complementary directions: if one starts from $\Phi_{+}$, one can stay forever in $\Phi_{+}$ only evolving forward in time. In other words one chooses the temporal orientation, fixes the signature of $\Phi_{ \pm}$, and cannot go backward.

\section{REVERSED HARMONIC OSCILLATOR: REMARKABLE RESULTS}

We consider the family of operators [20]

$$
\hat{V}_{\lambda}=\exp \left\{\frac{\lambda}{2}(\hat{x} \hat{p}+\hat{p} \hat{x})\right\} .
$$

In a system of measurement where $\hbar=1$, we have $[\hat{x}, \hat{p}]=i$, so

$$
\hat{V}_{\lambda} \phi(x)=e^{-i \frac{\lambda}{2}} \phi\left(e^{-i \lambda} x\right)
$$

whence

$$
\hat{V}_{\lambda} \hat{x} \hat{V}_{\lambda}^{-1}=e^{-i \lambda} \hat{x}
$$

and

$$
\hat{V}_{\lambda} \hat{p} \hat{V}_{\lambda}^{-1}=e^{i \lambda} \hat{p}
$$

If we recall the Hamiltonian in Eq. (8)

$$
H_{\mathrm{HO}}:=\frac{\hat{p}^{2}}{2}+\frac{\gamma^{2} \hat{x}^{2}}{2},
$$

it is easy to see that

$$
\hat{V}_{ \pm \frac{\pi}{4}} H \hat{V}_{ \pm \frac{\pi}{4}}^{-1}= \pm i H_{\mathrm{HO}},
$$

and we can transform the results we already know for the $\mathrm{HO}$ in results for the RHO:

$$
\begin{aligned}
E_{n}^{\mathrm{HO}} & =\gamma\left(n+\frac{1}{2}\right), \quad E_{n}=i E_{n}^{\mathrm{HO}} \in \mathbb{C}, \\
\psi_{n}^{\mathrm{HO}} & =\left(\frac{\gamma}{\pi}\right)^{1 / 4}\left(2^{n} n !\right)^{-1 / 2} e^{-\frac{\gamma}{2} x^{2}} H_{n}(\sqrt{\gamma} x), \\
f_{n}^{ \pm} & =\hat{V}_{ \pm \frac{\pi}{4}}^{-1} \psi_{n}^{\mathrm{HO}} \in S^{\times}(\mathbb{R}) .
\end{aligned}
$$

\section{A. Unitary transformation: From $(u, v)$ to $(x, p)$ framework}

One passes from the HO to the RHO through the operator $\hat{V}_{ \pm \frac{\pi}{4}}$, but one can also pass from $H(\hat{u}, \hat{v})$ to $H(\hat{x}, \hat{p})$, i.e., from the damped motion to the RHO, through a canonical transformation and find a relation between the spectra of these two Hamiltonians.

The canonical transformation from $(u, v)$ to $(x, p)$ is generated by the generating function

$$
S(x, u)=\frac{\gamma}{2} x^{2}-\sqrt{2 \gamma} x u+\frac{1}{2} u^{2},
$$

with $p=\frac{\partial S}{\partial x}, v=-\frac{\partial S}{\partial u}$.

We define the unitary transformation

$$
\mathcal{U}: \mathcal{L}^{2}(\mathbb{R}) \longrightarrow \mathcal{L}^{2}(\mathbb{R})
$$

such that

$$
f(u) \longrightarrow(\mathcal{U} f)(x)=\tilde{C} \int_{\mathbb{R}} f(u) e^{i S(x, u)} d u,
$$

with $\tilde{C}:=e^{-i \frac{\pi}{8}} \sqrt[4]{\frac{\gamma}{2 \pi^{2}}}$ and we can prove that $\mathcal{U}$ is unitary by demonstrating that

$$
|\tilde{C}|^{2} \int_{\mathbb{R}} e^{i\left[S(x, u)-S\left(x^{\prime}, u\right)\right]} d u=\delta\left(x-x^{\prime}\right) .
$$

To get a relation of quasiorthogonality and quasicompleteness for the resonances, we need to understand the nature of 
the operator $\hat{V}_{\lambda}$. It acts almost like the evolution operator $U$ in Eq. (25), with a complex (instead of real) exponential, but this is enough only to say that $\hat{V}_{\lambda}$ is unitary for pure imaginary $\lambda$, not for every $\lambda \in \mathbb{C}$. In fact, for a generic $\lambda=\omega+i \gamma$, where $\omega, \gamma \in \mathbb{R}$, one has

$$
\begin{aligned}
\left\langle\hat{V}_{\lambda} \phi \mid \hat{V}_{\lambda} \psi\right\rangle & =\int_{\mathbb{R}} d x\left[e^{\frac{\gamma-i \omega}{2}} \phi\left(e^{\gamma-i \omega} x\right)\right]^{*} e^{\frac{\gamma-i \omega}{2}} \psi\left(e^{\gamma-i \omega} x\right) \\
& =e^{i \omega} \int_{\mathbb{R}} d x[\phi(x)]^{*} \psi(x)=e^{i \omega}\langle\phi \mid \psi\rangle .
\end{aligned}
$$

Therefore it is not surprising that $f_{n}^{ \pm}$are only proportional to $\mathcal{U}\left[f_{n}^{ \pm}(u)\right](x)$ and not exactly equal. In fact

$$
f_{n}^{ \pm}(x)=e^{i n \frac{\pi}{4}}(2 \pi)^{ \pm \frac{1}{4}} \mathcal{U}\left[f_{n}^{ \pm}(u)\right](x)
$$

Nevertheless, we achieve the same relation of quasiorthogonality and quasicompleteness we had before:

$$
\begin{gathered}
\left\langle f_{n}^{ \pm}(x) \mid f_{m}^{\mp}(x)\right\rangle=\delta_{n m}, \\
\sum_{n=0}^{+\infty}\left[f_{n}^{ \pm}(x)\right]^{*} f_{n}^{\mp}\left(x^{\prime}\right)=\delta\left(x-x^{\prime}\right) .
\end{gathered}
$$

Moreover

$$
\left[f_{n}^{ \pm}(x)\right]^{*}=f_{n}^{\mp}(x) .
$$

Recalling Eqs. (15) and (16) and the meaning of the inverse Fourier transform for the damped motion represented by $\hat{H}(\hat{u}, \hat{v})$ (the inverse Fourier transform coincides with the time-reversal operator $T$ in that system), one has $T=C$, where $C$ is the complex-conjugation operator, as shown in [20].

We want to find $\chi^{E}$ such that

$$
H \chi^{E}=E \chi^{E}
$$

From [20] we get the complete derivation of the following solutions:

$$
\begin{aligned}
\chi_{+}^{E}(x) & =\frac{\tilde{C}}{\sqrt{2 \pi \gamma}} i^{\frac{\nu+1}{2}} \Gamma(\nu+1) D_{-v-1}(-\sqrt{-2 \gamma i} x), \\
\chi_{-}^{E}(x) & =\chi_{+}^{E}(-x),
\end{aligned}
$$

where here $v=-\left(i \frac{E}{\gamma}+\frac{1}{2}\right)$ and

$$
D_{\nu}(z):=\frac{e^{-\frac{z^{2}}{4}}}{\Gamma(-v)} \int_{\mathcal{R}} \xi_{ \pm}^{-v-1} e^{\mp z \xi-\frac{1}{2} \xi^{2}} d \xi
$$

is a Whittaker function [31].

If one remembers Eq. (18), one knows that the set of eigenfunctions is not complete yet. In fact, the two families of functions $\eta_{ \pm}^{E}(x):=\left(\mathcal{U} \breve{F}\left[\psi_{ \pm}^{-E}\right]\right)(x)$ are still missing, and we obtain

$$
\begin{aligned}
H \eta_{ \pm}^{E} & =-E \eta_{ \pm}^{E} \\
\eta_{+}^{E}(x) & =\frac{\tilde{C}}{\sqrt{2 \pi \gamma}} i^{\frac{v+1}{2}} \Gamma(-v) D_{\nu}(-\sqrt{2 \gamma i} x), \\
\eta_{-}^{E}(x) & =\eta_{+}^{E}(-x) .
\end{aligned}
$$

We observe that

$$
\eta_{ \pm}^{E}(x)=\left[\chi_{ \pm}^{E}(x)\right]^{*},
$$

which confirms that the time-reversal operator $T$ acts like the complex conjugation $C$.

From the corresponding properties satisfied by $\psi_{ \pm}^{E}(u)$ and from the unitary nature of $\mathcal{U}$ we have

$$
\begin{aligned}
& \sum_{ \pm} \int_{\mathbb{R}}\left[\chi_{ \pm}^{E}(x)\right]^{*} \chi_{ \pm}^{E^{\prime}}(x) d x=\delta\left(E-E^{\prime}\right), \\
& \sum_{ \pm} \int_{\mathbb{R}}\left[\chi_{ \pm}^{E}(x)\right]^{*} \chi_{ \pm}^{E}\left(x^{\prime}\right) d E=\delta\left(x-x^{\prime}\right), \\
& \sum_{ \pm} \int_{\mathbb{R}}\left[\eta_{ \pm}^{E}(x)\right]^{*} \eta_{ \pm}^{E^{\prime}}(x) d x=\delta\left(E-E^{\prime}\right), \\
& \sum_{ \pm} \int_{\mathbb{R}}\left[\eta_{ \pm}^{E}(x)\right]^{*} \eta_{ \pm}^{E}\left(x^{\prime}\right) d E=\delta\left(x-x^{\prime}\right) .
\end{aligned}
$$

At this point, we have all the tools we need to study the analytic properties of these four families of eigenfunctions. The outcome is that $\chi_{ \pm}^{E}(x)$ and $\eta_{ \pm}^{E}(x)$ have simple poles at $E=-E_{n}$ and $E=E_{n}$, respectively. Furthermore,

$$
\begin{aligned}
\operatorname{Res}\left[\chi_{ \pm}^{E}(x) ;-E_{n}\right] & =\frac{\tilde{C}}{\sqrt{2 \pi \gamma}} \frac{(-1)^{n}}{n !} i^{-\frac{n}{2}} D_{n}(\mp \sqrt{-2 \gamma i x}), \\
\operatorname{Res}\left[\eta_{ \pm}^{E}(x) ; E_{n}\right] & =\frac{\tilde{C}}{\sqrt{2 \pi \gamma}} \frac{(-1)^{n}}{n !} i^{\frac{n+1}{2}} D_{n}(\mp \sqrt{2 \gamma i} x) .
\end{aligned}
$$

In [31-33] one can find that $D_{n}(y)=2^{-\frac{n}{2}} e^{-\frac{y^{2}}{4}} H_{n}\left(\frac{z}{\sqrt{2}}\right)$. This, together with $H_{n}(-y)=(-1)^{n} H_{n}(y)$, allows us to obtain

$$
\operatorname{Res}\left[\chi_{ \pm}^{E}(x) ;-E_{n}\right] \propto f_{n}^{+}(x)
$$

and

$$
\operatorname{Res}\left[\eta_{ \pm}^{E}(x) ; E_{n}\right] \propto f_{n}^{-}(x) .
$$

Following Sec. V, we get $\Phi_{ \pm}$from the residues of the RHO eigenfunctions:

$$
\begin{aligned}
\mathcal{H} & =\mathcal{L}^{2}(\mathbb{R}), \\
\Phi & =S(\mathbb{R}), \\
\Phi_{-} & =\left\{\phi \in \Phi \mid f(E)=\left\langle\phi \mid \eta_{ \pm}^{E}\right\rangle \in \mathcal{H}_{-}^{2}\right\}, \\
\Phi_{+} & =\left\{\phi \in \Phi \mid f(E)=\left\langle\phi \mid \chi_{ \pm}^{E}\right\rangle \in \mathcal{H}_{+}^{2}\right\} .
\end{aligned}
$$

\section{B. Evolution operator acting like a semigroup}

We study waves $\phi_{ \pm} \in \Phi_{ \pm}$and the action of the evolution operator. We have $T=C$ and

$$
T\left(\Phi_{+}\right)=\Phi_{-} .
$$


Writing envelopes of $\phi_{ \pm}$in series of resonances, we obtain

$$
\begin{aligned}
\phi^{+}(x) & =\sum_{n=0}^{+\infty}\left\langle\phi^{+} \mid f_{n}^{+}\right\rangle^{*} f_{n}^{-}(x) \forall \phi^{+} \in \Phi_{+}, \\
\phi^{-}(x) & =\sum_{n=0}^{+\infty}\left\langle\phi^{-} \mid f_{n}^{-}\right\rangle^{*} f_{n}^{+}(x) \forall \phi^{-} \in \Phi_{-} .
\end{aligned}
$$

Thanks to the Gelfand-Maurin spectral theorem

$$
\phi^{+}(x)=\sum_{ \pm} \int_{\mathbb{R}} d E \chi_{ \pm}^{E}(x)\left\langle\phi^{+} \mid \psi_{ \pm}^{E}\right\rangle^{*}
$$

and

$$
\phi^{-}(x)=\sum_{ \pm} \int_{\mathbb{R}} d E \eta_{ \pm}^{E}(x)\left\langle\phi^{-} \mid \eta_{ \pm}^{E}\right\rangle^{*} .
$$

In conclusion, even in this case, the temporal evolution operator $U(t)=e^{-i H t}$ establishes a unitary group on $\mathcal{H}=$ $\mathcal{L}^{2}(\mathbb{R})$, and two semigroups:

$$
\begin{aligned}
& U_{+}(t): \Phi_{+} \longrightarrow \Phi_{+} \quad \forall t \geqslant 0 ; \\
& U_{-}(t): \Phi_{-} \longrightarrow \Phi_{-} \quad \forall t \leqslant 0 .
\end{aligned}
$$

Furthermore, if $\phi^{+}(x, 0) \in \Phi_{+}$then

$$
\phi^{+}(x, t)=\sum_{n} e^{-\gamma(n+1 / 2) t}\left\langle\phi^{+} \mid f_{n}^{+}\right\rangle^{*} f_{n}^{-}(x),
$$

while, if $\phi^{-}(x, 0) \in \Phi_{-}$then

$$
\phi^{-}(x, t)=\sum_{n} e^{\gamma(n+1 / 2) t}\left\langle\phi^{-} \mid f_{n}^{-}\right\rangle^{*} f_{n}^{+}(x) .
$$

We stress again that we obtained an irreversible quantum theory by studying the action of $U$ on $\Phi_{ \pm}$as a semigroup. Time $t=0$ splits the evolution in two diametrically opposed directions, and it becomes the instant which separates two different dynamics.

\section{FUNCTIONS WITH COMPACT SUPPORT}

In this section we examine a function set in $\Phi_{+}$. We start working in the $(u, v)$ representation, where $H(\hat{u}, \hat{v})=$ $-\frac{\gamma}{2}[\hat{u} \hat{v}+\hat{v} \hat{u}]$. We analyze the evolution in $(u, v)$ and $(x, p)$ planes. In $(x, p)$ the Hamiltonian is $H=\frac{\hat{p}^{2}}{2}-\frac{\gamma^{2} \hat{x}^{2}}{2}$ (we fix $\gamma=1$ hereafter).

\section{A. Wave packets in $(u, v)$ plane}

We previously proved that $\Phi_{+}$and $\Phi_{-}$coincide with $\mathcal{D}$ and $\mathcal{Z}$, respectively. We choose the forward orientation of the temporal arrow, so we focus our attention on the triplet

$$
\Phi_{+} \subset \mathcal{H} \subset \Phi_{+}^{\times},
$$

that is, $\mathcal{D} \subset \mathcal{L}^{2}(\mathbb{R}) \subset \mathcal{D}^{\times}$.

Let us consider the family of functions

$$
\phi_{\epsilon}(u)=\left\{\begin{array}{cl}
K_{\epsilon} \exp \left[\frac{1}{\left(\frac{u}{\epsilon}\right)^{2}-1}\right] & |u|<\epsilon, \\
0 & |u| \geqslant \epsilon,
\end{array}\right.
$$

where $\epsilon>0$ and $K_{\epsilon}$ is such that $\left\|\phi_{\epsilon}\right\|_{2}=1$, i.e., $\left[\int_{\mathbb{R}}\left|\phi_{\epsilon}(u)\right|^{2} d x\right]^{\frac{1}{2}}=1$ (see Fig. 1). $\phi_{\epsilon}(u)$ is a function of class

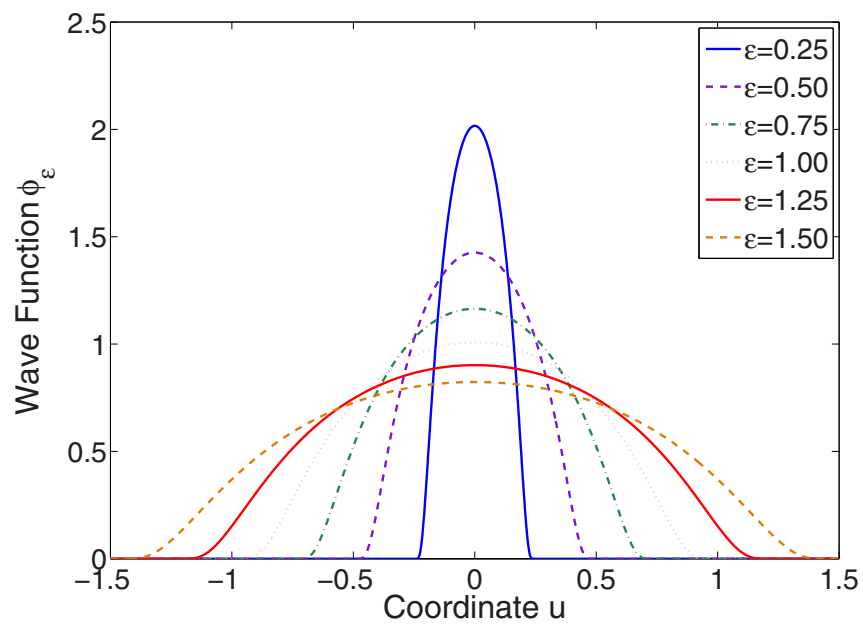

FIG. 1. Functions $\phi_{\epsilon}$ with compact support defined in Eq. (28) for several $\epsilon$ values.

$C^{\infty}(\mathbb{R})$, precisely

$$
\phi_{\epsilon}(u) \in \mathcal{D} \quad \forall \epsilon>0 .
$$

Starting from

$$
\sum_{n=0}^{\infty} f_{n}^{-}(u) f_{n}^{+}(w)=\delta(u-w),
$$

with $f_{n}^{-}(u)=\frac{(-1)^{n}}{\sqrt{n !}} \frac{d^{n}}{d u^{n}} \delta(u)$ and $f_{n}^{+}(u)=\frac{u^{n}}{\sqrt{n !}}$, we have

$$
\begin{aligned}
\phi_{\epsilon}(u) & =\int_{\mathbb{R}} d w \delta(u-w) \phi_{\epsilon}(w) \\
& =\int_{\mathbb{R}} d w \sum_{n=0}^{\infty} f_{n}^{-}(u) f_{n}^{+}(w) \phi_{\epsilon}(w) \\
& =\sum_{n=0}^{\infty} f_{n}^{-}(u)\left\langle\phi_{\epsilon} \mid f_{n}^{+}\right\rangle,
\end{aligned}
$$

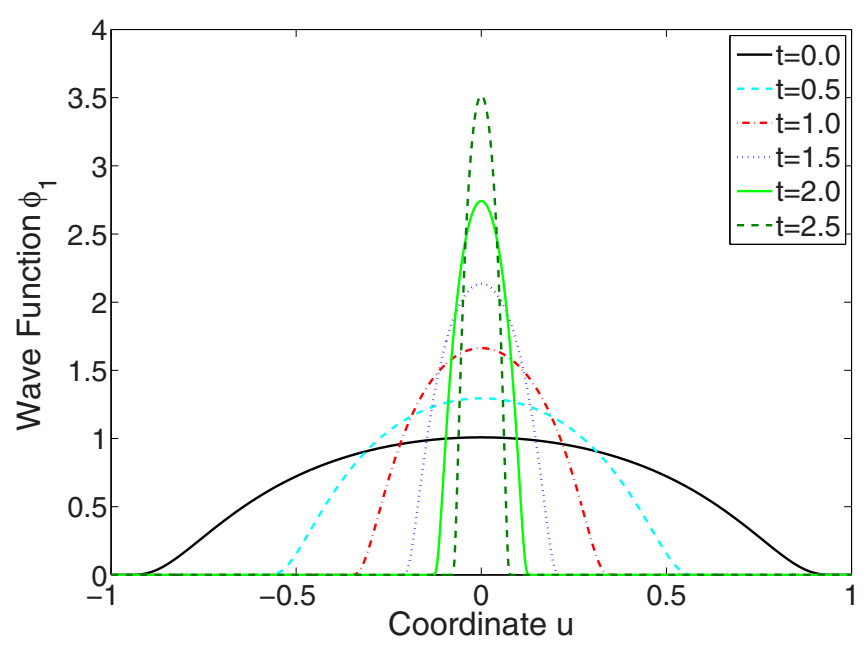

FIG. 2. One-dimensional evolution of $\left|\phi_{1}(u, t)\right|$ [Eq. (28) with $\epsilon=1]$. 


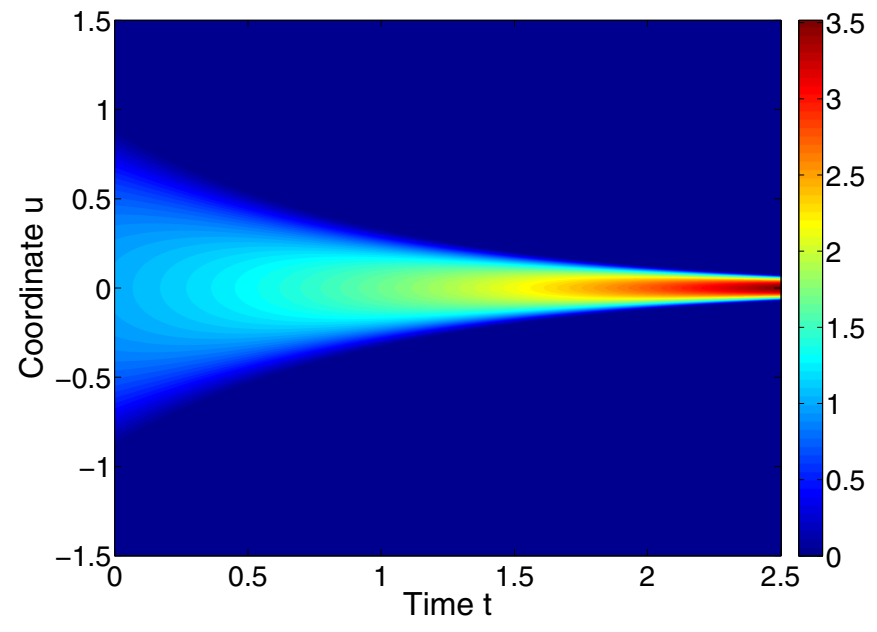

FIG. 3. Evolution of $\left|\phi_{1}(u, t)\right|$ [Eq. (28) with $\epsilon=1$ ].

since $\phi_{\epsilon} \in \mathcal{D}$. In deriving Eq. (29), as discussed in Sec. V and in [20], the residue theorem allows us to swap the integral and the summation. This is not valid for general functions in $\Phi$ not belonging to $\Phi_{+}$. We define the $N$-order background function as

$$
\phi_{N}^{\mathrm{BG}}(u, t):=\phi(u, t)-\sum_{n=0}^{N} f_{n}^{-}(u)\left\langle U(t) \phi \mid f_{n}^{+}\right\rangle^{*} \in \Phi^{\times},
$$

consequently

$$
\phi(u, t)=\sum_{n=0}^{N} f_{n}^{-}(u)\left\langle U(t) \phi \mid f_{n}^{+}\right\rangle^{*}+\phi_{N}^{\mathrm{BG}}(u, t) \quad \forall \phi \in \Phi .
$$

For $\phi \in \Phi_{+}, \phi_{N \rightarrow+\infty}^{\mathrm{BG}}=0$ and $U(t)$ acts as a semigroup. The evolution is a superposition of exponentially decaying functions. In contrast, for $\phi \notin \Phi_{+}, \phi_{N \rightarrow+\infty}^{\mathrm{BG}}$ does not converge

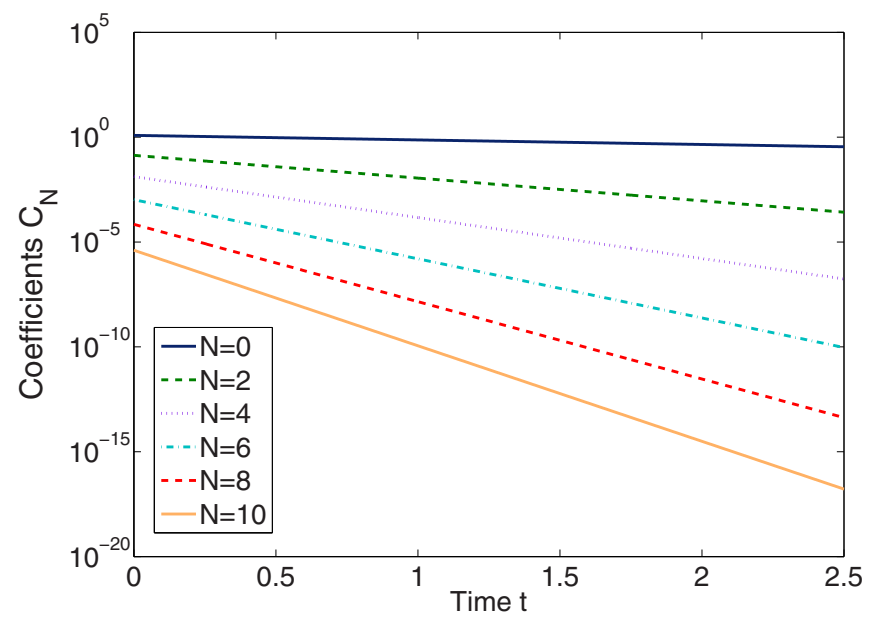

FIG. 4. Numerically calculated projections $C_{N}(t):=$ $\left\langle U(t) \phi_{1} \mid f_{N}^{+}\right\rangle^{*}$ on the $N$-order resonances of a function with compact support [Eq. (28) with $\epsilon=1]$ in the $(u, v)$ representation, in a semilogarithmic scale.

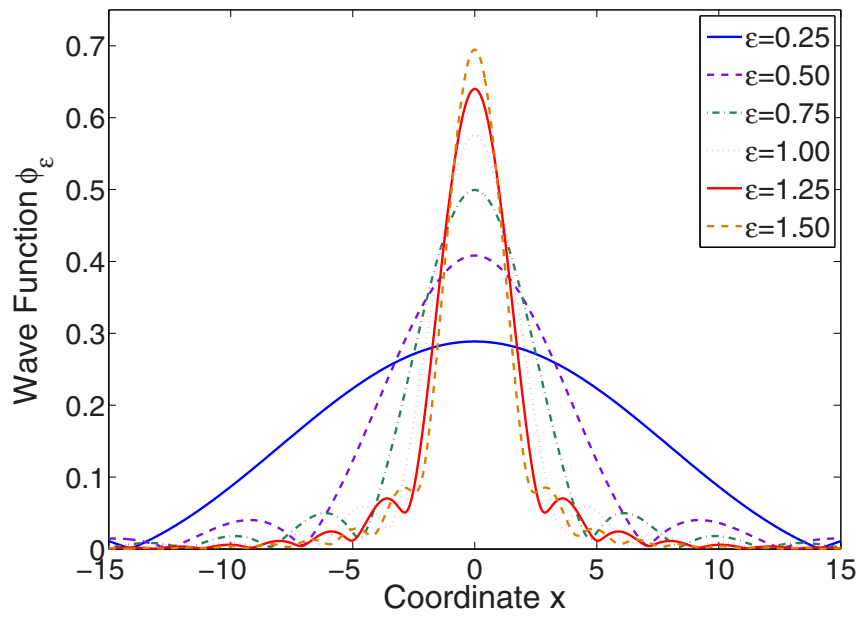

FIG. 5. Transformed $\phi_{\epsilon}$ [Eq. (30) for various $\left.\epsilon\right]$.

and the evolution includes nonexponentially decaying components.

We numerically simulate the Schrödinger equation $i \frac{\partial \psi}{\partial t}=$ $H \psi$ for the Hamiltonian $H=i \gamma\left(u \frac{\partial}{\partial u}+\frac{1}{2}\right)($ with $\gamma=1)$, with initial condition $\psi(u, t=0)=\phi_{\epsilon}(u)$. Figures 2 and 3 show the resulting "focusing" evolution.

Figure 4 reports the evolution of the coefficients $C_{N}(t):=$ $\left\langle U(t) \phi_{1} \mid f_{N}^{+}\right\rangle^{*}$. These brackets exponentially decay, with quantized decay rates. Into a semilogarithmic scale, the decay rates correspond to straight lines with different slopes.

\section{B. Wave packets in $(x, p)$ plane}

We pass from the $(u, v)$ to $(x, p)$ by the unitary transformation $\mathcal{U}$ :

$$
\begin{aligned}
\phi_{\epsilon}(x) & =\mathcal{U}\left[\phi_{\epsilon}(u)\right](x)=\sum_{n=0}^{\infty} \mathcal{U}\left[f_{n}^{-}(u)\right](x)\left\langle\mathcal{U} \phi_{\epsilon} \mid \mathcal{U} f_{n}^{+}\right\rangle \\
& =\sum_{n=0}^{\infty} f_{n}^{-}(x)\left\langle\phi_{\epsilon} \mid f_{n}^{+}\right\rangle,
\end{aligned}
$$

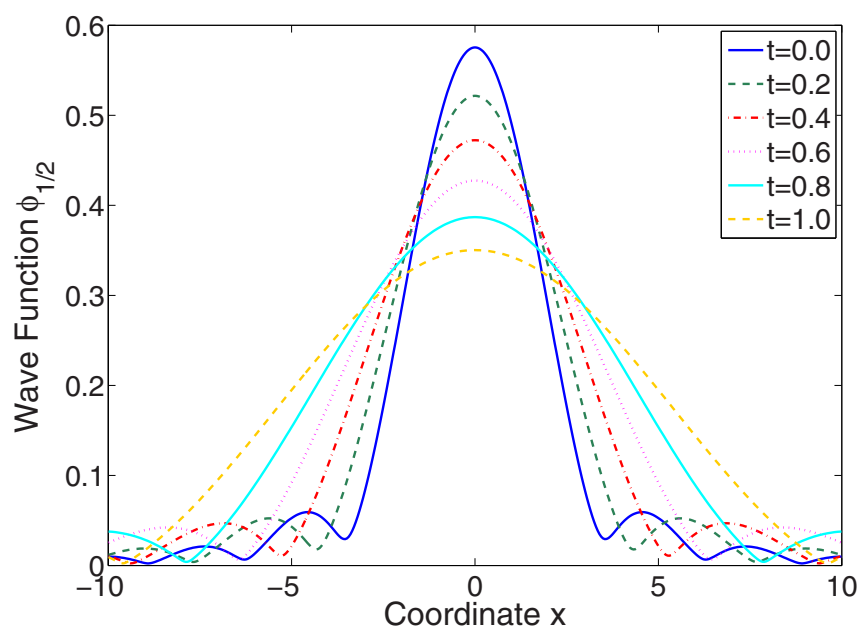

FIG. 6. One-dimensional evolution of a transformed function with compact support [Eq. (30), $\epsilon=1 / 2$ ] with a RHO potential. 


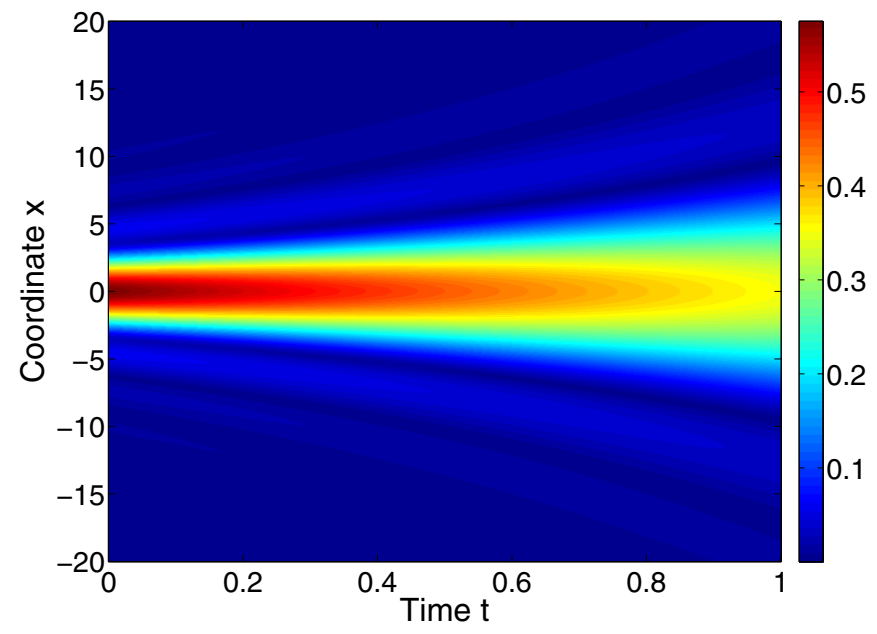

FIG. 7. Evolution of a transformed function with compact support [Eq. (30), $\epsilon=1 / 2$ ] with a RHO potential.

with $f_{n}^{ \pm}(x)=\hat{V}_{ \pm \frac{\pi}{4}}^{-1} \psi_{n}^{\mathrm{HO}}(x)$.

We numerically analyze the transformed functions. In Fig. 5, one can see several $\left(\mathcal{U} \phi_{\epsilon}\right)(x)$. We remark that functions $\phi_{\epsilon}$, which have compact support in $(u, v)$, do not have compact support in the $(x, p)$ phase plane.

We numerically study the evolution of wave packets in $(x, p)$. We solve numerically $i \frac{\partial \psi}{\partial t}=H \psi$ with initial condition $\psi(x, t=0)=\left(\mathcal{U} \phi_{\epsilon}\right)(x)$ and a RHO potential. Figures 6 and 7 show the resulting "defocusing" evolution.

\section{GAUSSIAN FUNCTION}

We examine the Gelfand triplet in Eq. (7) defined in Secs. V and VI, in the case of the Gaussian function as an element of the Hilbert space but not belonging to either $\Phi_{+}$or $\Phi_{-}$(see Fig. 8). For this kind of function, the expansion in Gamow states must be truncated and completed by an additional background function, not decaying exponentially, as discussed in Sec. VII A. We illustrate theoretically and numerically the properties of the background function, specifically studying a Gaussian function $\phi(u)=\frac{e^{-\frac{u^{2}}{2}}}{\sqrt[4]{\pi}}$ and its transformed $\mathcal{U}[\phi](x)$. We analyze the evolution both in $(u, v)$ and $(x, p)$ planes.

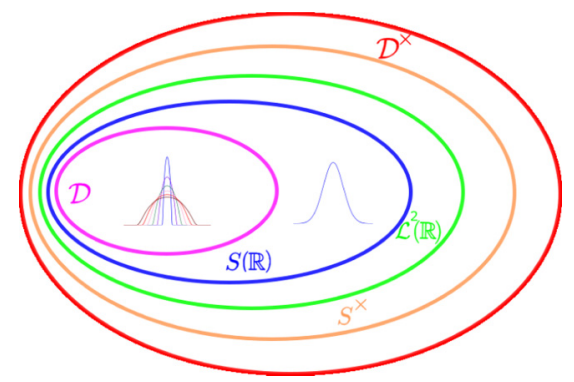

FIG. 8. Pictorial representation of Gelfand triplet defined in Eq. (7). Here $\Phi_{+} \equiv \mathcal{D}, \Phi \equiv S(\mathbb{R})$, and $\mathcal{H} \equiv \mathcal{L}^{2}(\mathbb{R})$. One can get a Euler-Venn diagram also for the triplet in Eq. (6) by replacing $\Phi_{+}$ with $\Phi_{-}$and $\mathcal{D}$ with $\mathcal{Z}$

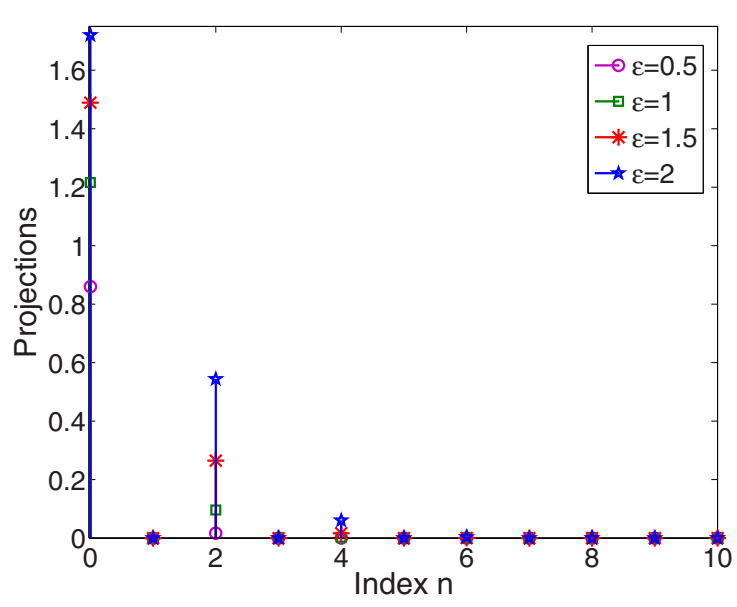

FIG. 9. Projections $\left\langle\phi_{1} \mid f_{n}^{+}\right\rangle^{*}$ of a function with compact support [defined in Eq. (28), $\epsilon=1$ ].

\section{A. Background function}

Let us define a normalized Gaussian function as

$$
\phi(u)=\frac{1}{\sqrt[4]{\pi}} e^{-\frac{u^{2}}{2}} \in S(\mathbb{R}) .
$$

$\phi(u)$ does not belong to $\mathcal{D}$ or to $\mathcal{Z}$ because the Fourier transform of a Gaussian function is still a Gaussian function and $\mathcal{D} \cap \mathcal{Z}=\emptyset$. Since $\sum_{n=0}^{+\infty} f_{n}^{+}(u) f_{n}^{-}(w)=\delta(u-w)$, we have

$$
\phi(u)=\int_{\mathbb{R}} d w \sum_{n=0}^{+\infty} f_{n}^{-}(u) f_{n}^{+}(w) \phi(w) \quad \forall \phi \in \Phi .
$$

The integral and the summation in Eq. (32) cannot be swapped in general, at variance with the case of $\phi_{\epsilon}(u)$ previously considered. Without loss of generality, we write

$$
\phi(u)=\sum_{n=0}^{N} f_{n}^{-}(u)\left\langle\phi \mid f_{n}^{+}\right\rangle^{*}+\phi_{N}^{\mathrm{BG}}(u),
$$

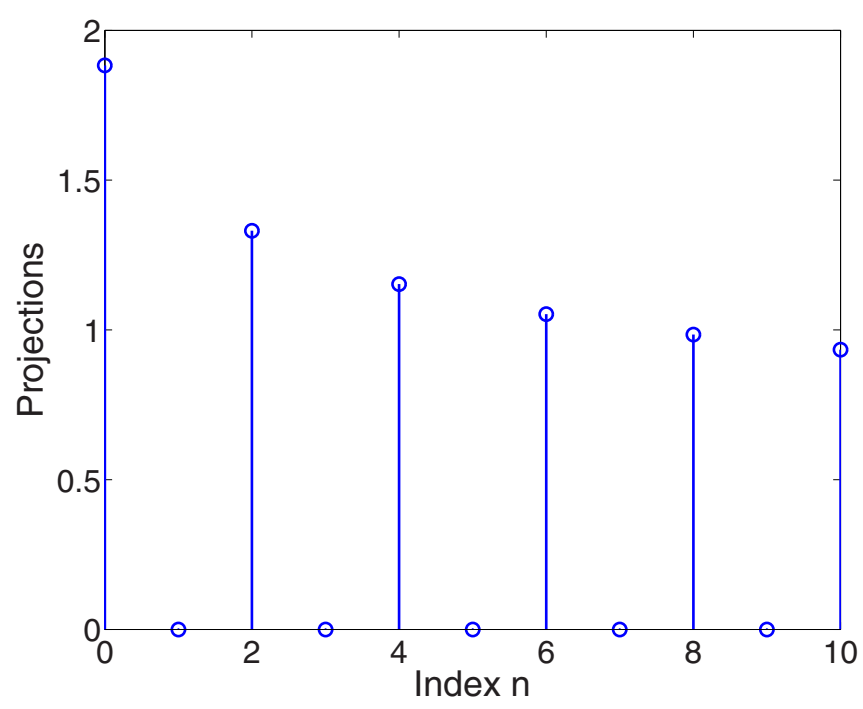

FIG. 10. Projections $\left\langle\phi \mid f_{n}^{+}\right\rangle^{*}$ of the Gaussian function. 


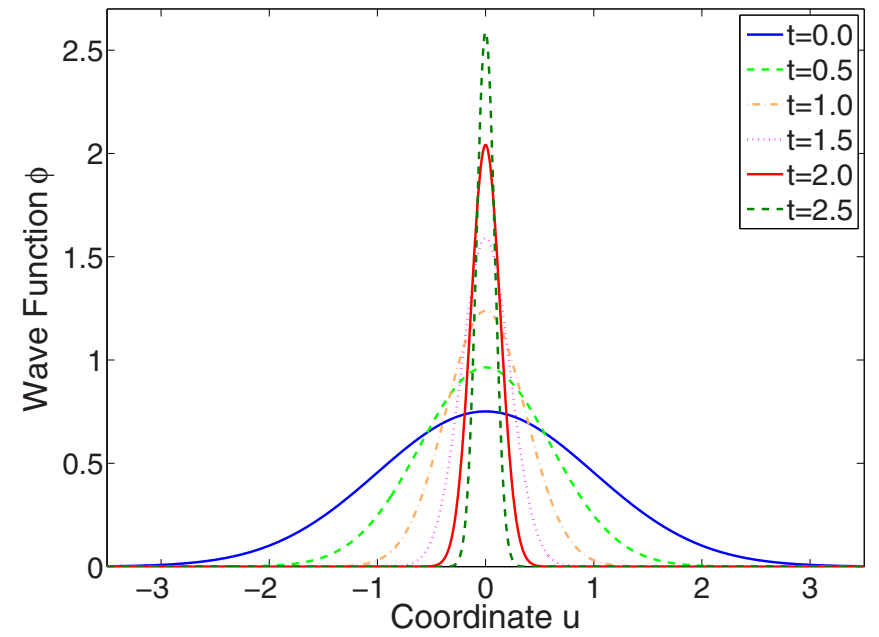

FIG. 11. One-dimensional evolution of the Gaussian wave $\phi(u)=\frac{e^{-\frac{u^{2}}{2}}}{\sqrt[4]{\pi}}$.

where $\phi_{N}^{\mathrm{BG}}(u)$ is the $N$-order background.

Since $\phi(u)=\phi(-u)$, while $f_{n}(u)=-f_{n}(-u)$ for odd $n$, we have

$$
\left\langle\phi \mid f_{n}^{+}\right\rangle=\left\{\begin{array}{cc}
\frac{2^{\frac{n+1}{2}} \Gamma\left(\frac{n+1}{2}\right)}{\sqrt[4]{\pi} \sqrt{n !}} & \text { for even } n \\
0 & \text { for odd } n
\end{array}\right.
$$

Both $\left\langle\phi \mid f_{n}^{+}\right\rangle$and $\left\langle\phi_{\epsilon} \mid f_{n}^{+}\right\rangle$decrease for even $n$, but the Gaussian $\left\langle\phi \mid f_{n}^{+}\right\rangle$decays much more slowly, as one can see by comparing Figs. 9 and 10. However, this is not a mathematical proof of the existence of the background. The presence of the background is proved through the study of the initial datum: $\phi_{\epsilon}(u)$ is an initial state that is composed only by a discrete sum of resonances without any component in the continuum, because it belongs to $\mathcal{D}$; in contrast, $\phi(u)$ is an initial state with a component of continuous radiation that is the background.

We analyze the evolved $N$-order background wave for Gaussian initial data. We want to study its limit as $N \rightarrow+\infty$.

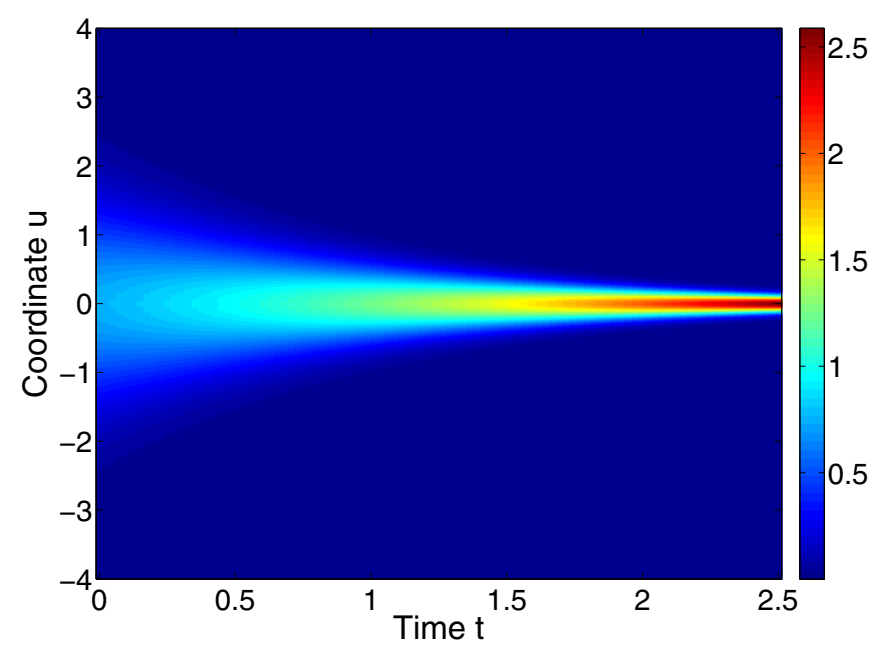

FIG. 12. Evolution of the Gaussian wave $\phi(u)=\frac{e^{-\frac{u^{2}}{2}}}{\sqrt[4]{\pi}}$.

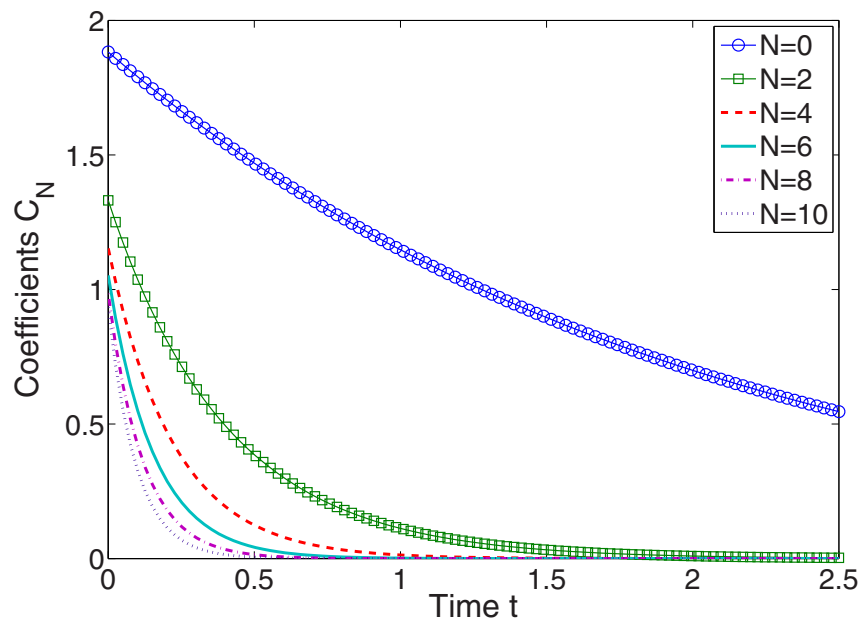

FIG. 13. Evolution of the projections $C_{N}(t):=\left\langle U(t) \phi \mid f_{N}^{+}\right\rangle^{*}$ on the $N$-order resonances of the Gaussian wave $\phi(u)=\frac{e^{-\frac{u^{2}}{2}}}{\sqrt[4]{\pi}}$ in the $(u, v)$ representation, in a linear scale.

We have

$$
\begin{aligned}
\phi_{N}^{\mathrm{BG}}(u, t) & =\phi(u, t)-\sum_{n=0}^{N} f_{n}^{-}(u)\left\langle f_{n}^{+}|U(t)| \phi\right\rangle \\
& =U(t) \phi(u)-\sum_{n=0}^{N} f_{n}^{-}(u)\left\langle f_{n}^{+}\left|U(t)^{\times}\right| \phi\right\rangle \\
& =U(t) \phi(u)-\sum_{n=0}^{N} e^{-\frac{\gamma}{2}(2 n+1) t} f_{n}^{-}(u)\left\langle f_{n}^{+} \mid \phi\right\rangle .
\end{aligned}
$$

We notice that the limit as $N$ approaches infinity could diverge or not exist. This happens in most cases, and specifically for the Gaussian function. In fact, in Eq. (33), we can approximate the $\Gamma$ function

$$
\Gamma(z)=\sqrt{2 \pi} z^{z-\frac{1}{2}} e^{-z}\left[1+O\left(\frac{1}{z}\right)\right]
$$

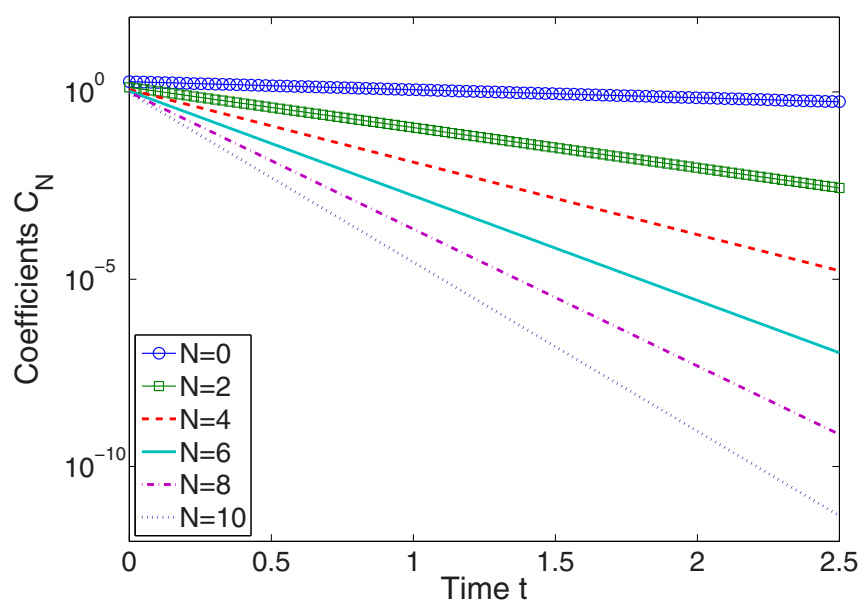

FIG. 14. Evolution of the projections on the $N$-order resonances $C_{N}(t):=\left\langle U(t) \phi \mid f_{N}^{+}\right\rangle^{*}$ of the Gaussian wave $\phi(u)=\frac{e^{-\frac{u^{2}}{2}}}{\sqrt[4]{\pi}}$ in the $(u, v)$ representation, in a semilogarithmic scale. 


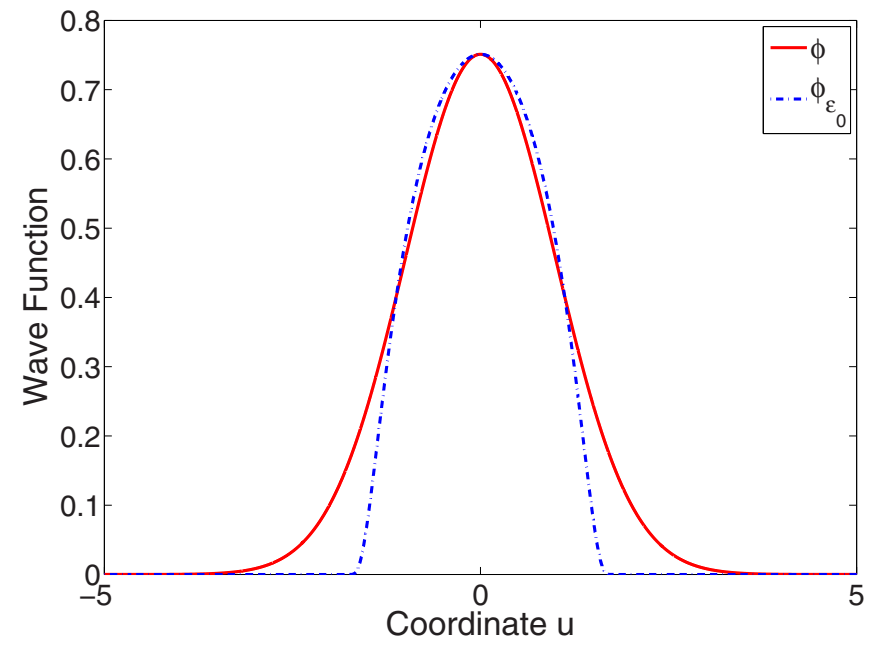

FIG. 15. Fit of the Gaussian function $\phi(u)=\frac{e^{-\frac{u^{2}}{2}}}{\sqrt[4]{\pi}}$ by the function $\phi_{\epsilon_{0}}(u)\left(\epsilon_{0}=1.802425\right)$.

and the factorial

$$
n !=\sqrt{2 \pi} n^{n+\frac{1}{2}} e^{-n}\left[1+O\left(\frac{1}{n}\right)\right]
$$

for large values of $z$ and $n$, thanks to the Stirling formula [31]. We find, for even $n$

$$
\left\langle\phi \mid f_{n}^{+}\right\rangle=\frac{2^{\frac{n+1}{2}} \Gamma\left(\frac{n+1}{2}\right)}{\sqrt[4]{\pi} \sqrt{n !}} \simeq \frac{2^{\frac{3}{4}}\left(1+\frac{1}{n}\right)^{\frac{n}{2}}}{\sqrt[4]{e^{2} n}} \simeq \frac{2^{\frac{3}{4}}}{\sqrt[4]{n}},
$$

hence $\left\langle\phi \mid f_{n}^{+}\right\rangle$approaches zero with order $\frac{1}{4}$, too slowly to let the series converge $\forall t \geqslant 0$ and $\forall u \in \mathbb{R}$, so the limit $N \rightarrow+\infty$ does not exist globally. This confirms that an expansion like Eq. (30) with an infinite number of GVs is meaningless for a Gaussian function, and a background term is needed.
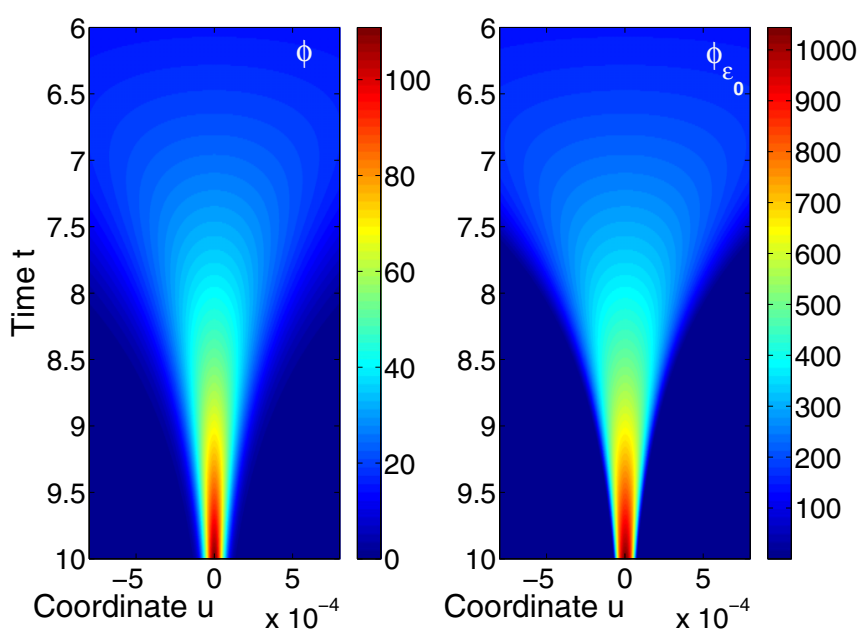

FIG. 16. Comparison of the Gaussian evolution $\phi(u, t)$ with $\phi_{\epsilon_{0}}(u, t) . \phi_{\epsilon_{0}}(u, t)$ focalizes without any loss or dispersion of energy, while the Gaussian presents a dispersive background (see also Fig. 17).

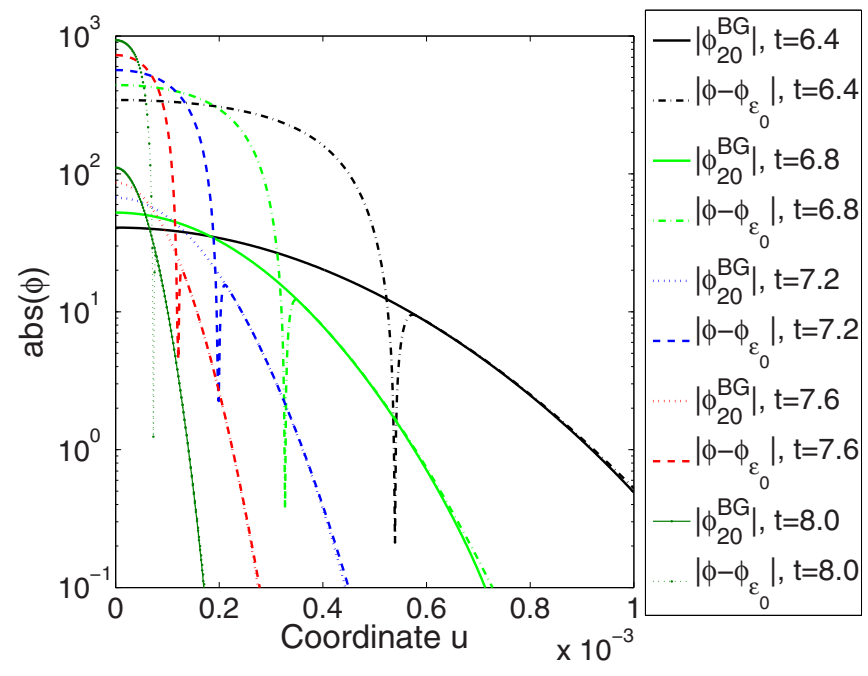

FIG. 17. We want to analyze if a region exists where $\phi_{N}^{\mathrm{BG}}(u, t) \simeq$ $\phi(u, t)-\phi_{\epsilon_{0}}(u, t)$. A comparison between $\phi_{20}^{\mathrm{BG}}(u, t)$ and $\phi(u, t)-$ $\phi_{\epsilon_{0}}(u, t)$ is here reported in semilogarithmic scale. These two wave packets are well overlapped on their borders.

\section{B. Evolution}

Figures 11 and 12 show a portrayal of the Gaussian function evolution. In Figs. 13 and 14 one can observe the decay of the coefficients.

The $(u, v)$ phase space remains the simplest configuration for numerical tests of the theory. Since one runs into a high computational complexity when analyzing the background evolution, we chose the $(u, v)$ phase space to compare the evolution of a Gaussian function with a specific $\phi_{\epsilon}$. We best fit the normalized Gaussian function by a function $\phi_{\epsilon}$ in order to compare the background function with the difference between these two waves. Figure 15 shows $\phi(u)$ and its best fit by $\phi_{\epsilon}(u)$, obtained for $\epsilon=\epsilon_{0}=1.802425$. Figure 16 compares the calculated evolution of $\phi(u)$ and $\phi_{\epsilon_{0}}$. We should see the dispersive component that occurs on the boundaries of the

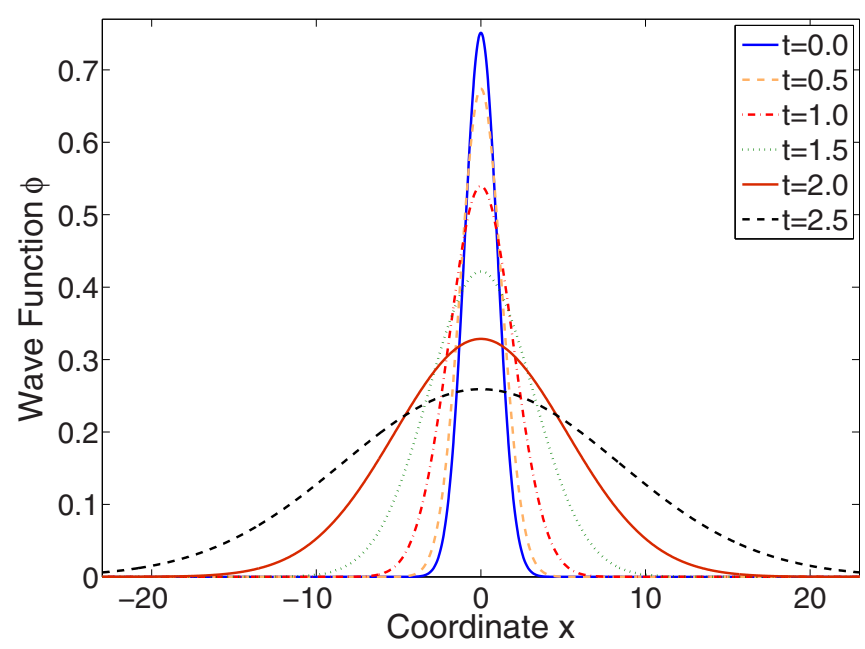

FIG. 18. One-dimensional evolution of the Gaussian wave $\phi(x)=\frac{e^{-\frac{x^{2}}{2}}}{\sqrt[4]{\pi}}$ under a RHO potential. 


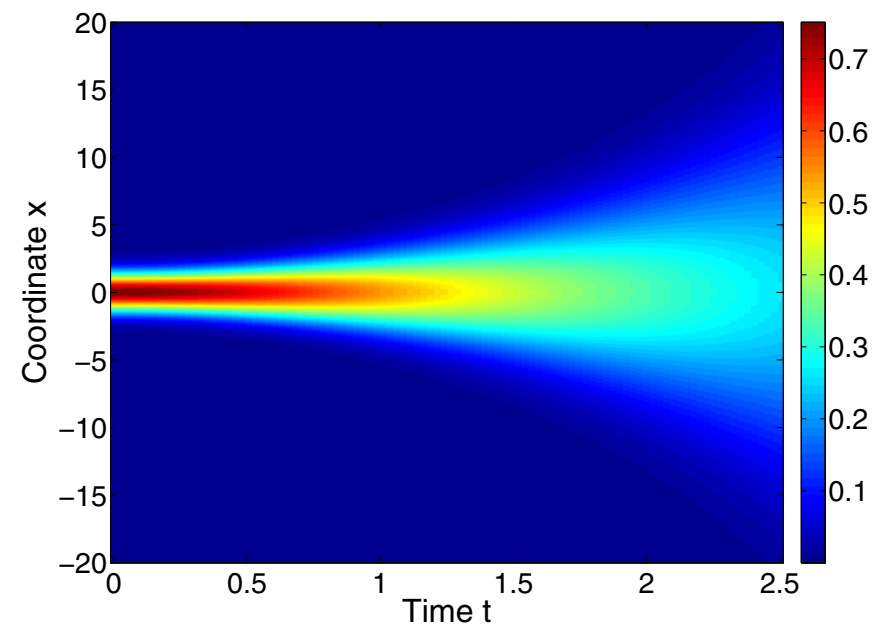

FIG. 19. Evolution of the wave $|\phi|$ with initial condition $\phi(x)=$ $\frac{e^{-\frac{x^{2}}{2}}}{\sqrt[4]{\pi}}$.

Gaussian evolution. However, appreciating the dispersive behavior is difficult in a linear scale; we report a comparison between $\phi_{20}^{\mathrm{BG}}$ and $\phi-\phi_{\epsilon_{0}}$ in Fig. 17 in a semilogarithmic scale. The continuous lines represent the Gaussian background for several time values, while the dashed lines give the difference between the Gaussian and the function with compact support. One can now see without difficulty that the outlines on the boundaries are well overlapped, so the long time evolution of a Gaussian background, that is, the dispersive tail of a function not belonging to $\Phi_{+}$, can be approximated to rest between the function we are studying and an appropriately chosen function $\phi_{\epsilon}$.

We want to now complete our analysis by considering the $(x, p)$ system. By the transformation $\mathcal{U}$ we see that $\phi(x)=$ $[\mathcal{U} \phi(u)](x)$ is a Gaussian function anyway, because

$$
[\mathcal{U} \phi(u)](x)=\pi^{-\frac{1}{4}} \tilde{C} \int_{\mathbb{R}} e^{-\frac{u^{2}}{2}} e^{i S(x, u)} d u=\left(\frac{\gamma}{\pi}\right)^{\frac{1}{4}} e^{-\frac{\gamma}{2} x^{2}} .
$$

The focusing dynamics in the $(u, v)$ space corresponds to a defocusing propagation in the $(x, p)$ space as shown in Figs. 18 and 19.

\section{CONCLUSIONS}

We reviewed the basic theorems and the mathematical properties underlying the time-asymmetric formulation of quantum mechanics with specific reference to the reversed harmonic oscillator. We considered the propagation of a wave packet in the reversed harmonic oscillator within the rigged-Hilbert-space framework. We specifically analyzed the evolution of a class of functions with compact support in the $(u, v)$ phase space and the evolution of a normalized Gaussian function. For the functions with compact support we discussed the way the dynamics in the $(u, v)$ maps into the real $(x, p)$ space, and verify that the projections of a wave packet on Gamow states decay exponentially. We studied the mechanism of excitation of the background function for a Gaussian function that does not belong to $\Phi_{+}$. The Gaussian function cannot be expressed as an infinite linear combination of GVs, and the result is the excitation of a dispersive wave which does not decay exponentially.

In other words, for a RHO the temporal evolution is dominated by a sum of exponentially decaying states with quantized decay rates. Depending on the function class of the initial conditions, one can also observe the excitation of a nonexponentially decaying component, denoted as the background. These findings may be directly tested in the experiments by a proper shaping of the initial conditions. We believe that our results address some of the known concepts of the RHS approach to the dynamics of unstable systems in a way that may find direct application in designing tests of time-asymmetric quantum physics, in fields like Bose-Einstein condensation, superconductors, and photonics. Gamow vectors may also open novel possibilities in studying nonlinear waves and their reversibility properties from a fundamental point of view.

\section{ACKNOWLEDGMENTS}

We acknowledge fruitful discussions with M. C. Braidotti, M. Materassi, P. M. Santini, and P. Verrucchi. This work was made possible through the support of a grant from the John Templeton Foundation (Grant No. 58277).
[1] A. Bohm, Phys. Rev. A 60, 861 (1999).

[2] A. Bohm and N. L. Harshman, in Irreversibility and Causality Semigroups and Rigged Hilbert Spaces, Lectures Notes in Physics Vol. 504-504, edited by A. Bohm, H.-D. Doebner, and P. Kielanowski (Springer, Berlin, Heidelberg, 1998), pp. 179-237.

[3] A. Bohm, M. Gadella, and G. B. Mainland, Am. J. Phys. 57, 1103 (1989).

[4] I. Prigogine, F. Mayné, C. George, and M. D. Haan, Proc. Natl. Acad. Sci. U.S.A. 74, 4152 (1977).

[5] I. E. Antoniou and I. Prigogine, Physica A 192, 443 (1993).

[6] I. Antoniou, L. Dmitrieva, Y. Kuperin, and Y. Melnikov, Computers Math. Applic. 34, 399 (1997).

[7] S. Gentilini, M. C. Braidotti, G. Marcucci, E. DelRe, and C. Conti, Phys. Rev. A 92, 023801 (2015).

[8] S. Gentilini, M. C. Braidotti, G. Marcucci, E. Del Re, and C. Conti, Sci. Rep. 5, 15816 (2015).
[9] S. Longhi and S. M. Eaton, Opt. Lett. 41, 1712 (2016).

[10] M. Materassi, Entropy 17, 1329 (2015).

[11] M. Zanin, D. Papo, P. A. Sousa, E. Menasalvas, A. Nicchi, E. Kubik, and S. Boccaletti, Phys. Rep. 635, 1 (2016).

[12] D. Calvani, A. Cuccoli, N. I. Gidopoulos, and P. Verrucchi, Open Syst. Inf. Dyn. 20, 1340002 (2013).

[13] N. Perra, V. Zlatic, A. Chessa, C. Conti, D. Donato, and G. Caldarelli, Europhys. Lett. 88, 48002 (2009).

[14] Y. Y. Liu, J. J. Slotine, and A. L. Barabási, Nature 473, 167 (2011).

[15] U. G. Aglietti and P. M. Santini, Phys. Rev. A 89, 022111 (2014).

[16] G. Gamow, Z. Phys. 51, 204 (1928).

[17] G. Gamow, Nature 122, 805 (1928).

[18] M. C. Braidotti, S. Gentilini, and C. Conti, Opt. Express 24, 21963 (2016). 
[19] D. Chruściński, J. Math. Phys. 44, 3718 (2003).

[20] D. Chruściński, J. Math. Phys. 45, 841 (2004).

[21] C. G. Bollini, O. Civitarese, A. L. D. Paoli, and M. Rocca, J. Math. Phys. 37, 4235 (1996).

[22] O. Civitarese and M. Gadella, Phys. Rep. 396, 41 (2004).

[23] R. de la Madrid and M. Gadella, Am. J. Phys. 70, 626 (2002).

[24] M. Gadella and F. Gomez, Int. J. Theor. Phys. 42, 2225 (2003).

[25] G. Barton, Ann. Phys. 166, 322 (1986).

[26] E. Celeghini, M. Gadella, and M. A. del Olmo, J. Math. Phys. 57, 072105 (2016).
[27] V. Serov, A. Bychenkov, V. Derbov, and S. Vinitsky, Proc. SPIE 4002, 79 (2000).

[28] A. Gleason, J. Math. Mech. 6, 885 (1957).

[29] M. H. Stone, Ann. Math. 33, 643 (1932).

[30] G. C. Hegerfeldt, Phys. Rev. Lett. 72, 596 (1994).

[31] I. Gradshteyn and I. Ryzhik, Table of Integrals, Series and Products (Academic, New York, 1965).

[32] P. M. Morse and H. Feshbach, Methods of Theoretical Physics (McGraw-Hill, New York, 1953).

[33] M. Abramowitz and I. Stegun, Handbook of Mathematical Functions (Dover, New York, 1972). 